\title{
Designing a Multistage Reverse Logistics Network Problem by Hybrid Genetic Algorithm
}

\author{
Jeong-eun Lee \\ Waseda University, IPS \\ 808-0135, JAPAN \\ leeje@toki.waseda.jp
}

\author{
Mitsuo Gen \\ Waseda University, IPS \\ 808-0135, JAPAN \\ gen@waseda.jp
}

\author{
Kyong-gu Rhee \\ Dongeui University \\ 614-714, KOREA \\ rhee@deu.ac.kr
}

\begin{abstract}
We formulate a mathematical model of remanufacturing system as multistage reverse Logistics Network Problem (mrLNP) with minimizing the total costs for reverse logistics. The total costs for reverse logistics include shipping cost, fixed cost of opening the disassembly centers and processing centers and inventory holding cost at these centers over finite planning horizons. In this paper, we formulate the mrLNP model as a three stage logistics network model. For solving this problem, in the 1st and the 2nd stages, we propose a Genetic Algorithm (GA) with priority-based encoding method combined with a new crossover operator called as Weight Mapping Crossover (WMX). Additionally also a heuristic approach is applied in the 3rd stage where parts are transported from some processing centers to one manufacturer.
\end{abstract}

\section{Categories and Subject Descriptors}

G.2.3 [Mathematics of Computing]: DISCRETE MATHEMATICS-Applications; G.2.2 [Mathematics of Computing]: DISCRETE MATHEMATICS-Graph Theory

\section{General Terms}

Algorithms, Design.

\section{Keywords}

Genetic Algorithm; multistage reverse Logistics Network Problem; priority-based encoding method; Weight Mapping Crossover.

\section{INTRODUCTION}

Recycling problem can affect not only a cost performance of recycling of a company, but also whole strategy of the company including production strategy and purchase policy. To deal with this problem the government should treat it as a part of industry [1]. In this paper, we consider a complex reverse logistics problem (rLP). This rLP is combined with three reverse processes, i.e., retuning process, disassembly process and processing process. In the practical reverse logistics problems, the different parts should be assigned different processing processes based on the processing competence of the processing center. For this reason, the challenges of our study are both in the mathematical formulation and effective approach construction. Firstly, we

Copyright is held by the author/owner(s).

GECCO'08, July 12-16, 2008, Atlanta, Georgia, USA.

ACM 978-1-60558-130-9/08/07. formulate this reverse logistics problem as a multistage network model with considering the practical logistics constraints. Following, for solving this multistage logistics model, we introduced a priority-based GA approach, which was proposed with Gen and Cheng. [2]. Difference with Gen et al.'s prioritybased GA, includes the extension of priority-based encoding method to apply multistage logistics model with multi-parts assignment constraint in $1^{\text {st }}$ and $2^{\text {nd }}$ stage, and combine a heuristic approach in the $3^{\text {rd }}$ stage between the processing center to the manufacturer). Lastly, we compare our GA approach with Prüfer number-based GA (pnGA), which is most used approach in logistics models.

\section{MAHEMATICAL FORMULATION}

In this Section, we consider constituents, variables, and assumptions for formulating a multistage logistics network model.

We make the following assumptions of this mrLNP:

A1. We consider logistics network for treating single product.

A2. If one product is disassembled then two parts are obtained.

A3. The parts that are possible to disassemble are given.

A4. We consider the inventory factor at disassembly center and processing center over finite planning horizons.

A5. The demand of parts by manufacturer is known in advanced.

A6. The maximum capacities about four echelons are known: returning centers, disassembly centers, processing centers and manufacturer.

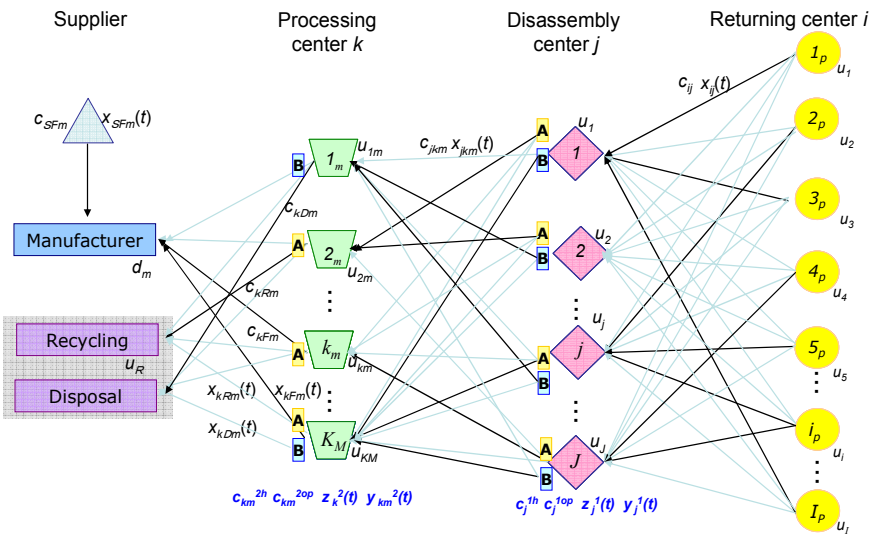

Figure 1. Multistage reverse logistics network model 
The mathematical model of the problem is:

$$
\begin{aligned}
\min z & =\sum_{t=0}^{T}\left[\sum_{i=1}^{I} \sum_{j=1}^{J} c_{i j} x_{i j}(t)+\sum_{j=1}^{J} \sum_{k=1}^{K} \sum_{m=1}^{M} c_{j k m} x_{j k m}(t)+\sum_{k=1}^{K} \sum_{m=1}^{M} c_{k F m} x_{k F m}(t)\right. \\
& -\sum_{k=1}^{K} \sum_{m=1}^{M} c_{k R m} x_{k R m}(t)+\sum_{k=1}^{K} \sum_{m=1}^{M} c_{k D m} x_{k D m}(t)+\sum_{m=1}^{M} c_{S F m} x_{S F m}(t) \\
& \left.+\sum_{j=1}^{J} c_{j}^{1 h} y_{j}^{1}(t)+\sum_{k=1}^{K} \sum_{m=1}^{M} c_{k m}^{2 h} y_{k m}^{2}(t)+\sum_{j=1}^{J} c_{j}^{1 o p} z_{j}^{1}+\sum_{k=1}^{K} c_{k}^{2 o p} z_{k}^{2}\right] \\
\text { s. t. } \quad & x_{S F m}(t)+\sum_{k=1}^{K} x_{k F m}(t) \geq d_{m}, \quad \forall m, t \\
& \sum_{j=1}^{J} x_{i j}(t)+y_{j}^{1}(t-1) \leq u_{j} z_{j}^{1}, \quad \forall j, t \\
& \sum_{k=1}^{K} x_{j k m}(t)+y_{k m}^{2}(t-1) \leq u_{k m} z_{k}^{2}, \quad \forall k, m, t \\
& x_{k R m} \quad(t) \leq u u_{R}, \quad \forall k, m, t \\
& \sum_{j=1}^{J} z_{j}^{1}(t) \leq J, \quad \forall t \\
& \sum_{k=1}^{K} z_{k}^{2}(t) \leq K, \quad \forall m, t \\
& x_{i j}(t), x_{j k m}(t), \quad x_{k F m}(t), x_{k R m}(t), x_{k D m}(t), x_{S F m}(t) \geq 0 \\
& z_{j}^{1}, z_{k}^{2} \in\{0,1\}
\end{aligned}
$$

\section{HYBRID GENETIC ALGORITHM}

We here adopt the priority-based encoding method developed Gen, Altiparmak and Lin [3]. Although this encoding had been successfully applied on shortest path problem and project scheduling problem, the difference of our approach comes from the facts that of special decoding and encoding procedures for transportation trees. The priority-based encoding method is an indirect approach. In this method, a gene in chromosome contains two kinds of information: the locus, the position of the gene within the structure of a chromosome, and the allele, the value the gene takes. The position of a gene is used to represent a node (source depot), and the value is used to represent the priority of the node for constructing a tree among candidates.

For a transportation problem, a chromosome consists of priorities of sources and depots to obtain transportation tree and its length is equal to total number of sources $\mathrm{m}$ and depots $\mathrm{n}$, i.e. $\mathrm{m}+\mathrm{n}$. The transportation tree corresponding with a given chromosome is generated by sequential arc appending between sources and depots. At each step, only one arc is added to tree selecting a source (depot) with the highest priority and connecting it to a repot (source) considering minimum cost.

For mrLNP, we use two priority-based encodings to represent the transportation trees on stages. This means that each chromosome in the population consists of two parts. While the first part (i.e. the first priority-based encoding) represents transportation tree between return centers and disassembly centers, the second part (i.e. the second priority-based encoding) represents transportation tree between disassembly centers and processing centers. The detailed encoding and decoding process of a chromosome consists of three main phase:

Phase 1: Coding process on 1st stage

Step 1.1: Priority-based encoding from returning center to disassembly center

Step 1.2: Decoding from returning center to disassembly center

Phase 2: Coding process on 2nd stage

Step 2.1: Priority-based encoding from disassembly center to processing center

Step 2.2: Decoding from disassembly center to processing center
Phase 3: A heuristic approach in the 3rd stage

1) Crossover Operator: We proposed a new crossover operator, weight mapping crossover (WMX). WMX is same as that of the conventional one-cut point crossover can generate two new paths that exchanged sub-route from two parents.

2) Mutation Operator: In this study, insertion mutation has been adopted. Insertion mutation selects a gene at random and inserts it in a random position.

\section{EXPERIMENT AND DISCUSSION}

We used using pnGA (Prüfer number-based GA) proposed by Syarif and Gen [4], to study the effectiveness of the developed GA with new encoding method (priGA). The number of returning centers changes between 4 and 30, number of disassembly centers and number of processing centers changed between 3-15, and 3-20, respectively. The transportation costs, demand of parts, capacitates of returning centers, disassembly processes, processing processes, recycles and manufacturer are randomly generated to provide realistic scenarios.

Numerical experiments demonstrated the efficiency and

\begin{tabular}{|c|c|c|c|c|c|c|c|c|c|}
\hline \multirow{2}{*}{$\begin{array}{c}\text { Problems } \\
\text { No. }\end{array}$} & \multirow{2}{*}{ stage } & \multirow{2}{*}{$\begin{array}{c}\text { no. of } \\
\text { constraints }\end{array}$} & \multirow{2}{*}{$\begin{array}{l}\text { no. of } \\
\text { shipping } \\
\text { variables }\end{array}$} & \multicolumn{3}{|c|}{ pnGA } & \multicolumn{3}{|c|}{ priGA } \\
\hline & & & & Best & Average & ACT & Best & Average & ACT \\
\hline \multirow[t]{4}{*}{1} & stage 1 & 1275 & 975 & 35650 & 35650 & 0.65 & 35650 & 35650 & 0.65 \\
\hline & stage 2 & & & 43200 & 43200 & 0.60 & 43200 & 43200 & 0.65 \\
\hline & stage 3 & & & 50100 & 50100 & - & 50100 & 50100 & - \\
\hline & total & & & 128950 & 128950 & 1.25 & 128950 & 128950 & 1.30 \\
\hline \multirow[t]{4}{*}{2} & stage 1 & 2505 & 2055 & 53795 & 54710 & 0.90 & 53490 & 54250 & 1.00 \\
\hline & stage 2 & & & 48270 & 50180 & 0.95 & 47370 & 49800 & 0.95 \\
\hline & stage 3 & & & 75500 & 75500 & - & 75500 & 75500 & - \\
\hline & total & & & 177565 & 180390 & 1.85 & 176360 & 179550 & 1.95 \\
\hline \multirow[t]{4}{*}{3} & stage 1 & 8745 & 7800 & 86300 & 88006 & 6.10 & 82920 & 85273 & 6.05 \\
\hline & stage 2 & & & 81480 & 81661 & 6.80 & 80730 & 83223 & 6.00 \\
\hline & stage 3 & & & 110050 & 110050 & - & 110050 & 110050 & - \\
\hline & total & & & 277830 & 279717 & 12.90 & 273700 & 278546 & 12.05 \\
\hline \multirow[t]{4}{*}{4} & stage 1 & 20430 & 18930 & 195895 & 199381 & 13.05 & 189765 & 193995 & 12.30 \\
\hline & stage 2 & & & 198915 & 205788 & 9.40 & 192900 & 199822.5 & 10.75 \\
\hline & stage 3 & & & 237675 & 237675 & - & 237675 & 237675 & - \\
\hline & total & & & 632485 & 642844 & 22.45 & 620340 & 631492.5 & 23.05 \\
\hline
\end{tabular}
effectiveness of the hybrid GA approach for solving the mrLNP problems. Although memory requirement for new representation was greater than pnGA, i.e., Prüfer number-based GA, only 2 digits for each stage in transportation problem, this representation got very important two advantages in the real world applications. One of them was that its implementation was very easy. Another one was that after genetic operators, always feasible solutions were obtained. Based on this study, it was seen that the hybrid priGA with WMX demonstrated the best performance according to solution quality.

Table. 1 Computational results with pnGA and priGA

\section{REFERENCES}

[1] Stock, J. K., 1992. Reverse logistics, White Paper, Council of Logistics Management, Oak Brook, IL.

[2] Gen, M. and Cheng, R. W., 1997. Genetic Algorithm and Engineering Design, Wiley, New York.

[3] Gen, M., Altiparmak, F. and Lin, L., 2006. A genetic algorithm for two-stage transportation problem using priority-based encoding, OR Spectrum, 28(3), 337-354.

[4] Syarilf, A. and Gen, M. 2003. Double Spanning Tree-based Genetic algorithm For Two Stage Transportation Problem, International Journal of Knowledge-Based Intelligent Engineering System, 7(4), 388-389. 